\title{
Immunisation against varicella in end stage and pre-end stage renal failure
}

Nicholas J A Webb, Margaret M Fitzpatrick, David A Hughes, Trevor J Brocklebank, Brian A Judd, Malcolm A Lewis, Robert J Postlethwaite, Patricia A Smith, Gerald Corbitt, on behalf of the Trans-Pennine Paediatric Nephrology Study Group

\begin{abstract}
Objectives-To investigate the seroconversion rate and duration of persistence of protective antibody titres after varicella immunisation in children with renal failure.

Design-32 children (25 end stage and 7 pre-end stage renal failure) were immunised using $2 \times 2000$ plaque forming unit doses of varicella vaccine 3 months apart. Varicella antibody titres were measured by enzyme linked immunosorbent assay.

Results-All children initially seroconverted after immunisation. At a mean follow up of 20.3 months, 23 of 28 had protective antibody titres, 4 children having died of unrelated causes. Two children required a third booster dose. 11 children underwent renal transplantation; $10 \mathrm{had}$ protective titres at the time of transplantation and, at a mean of 23.4 months after immunisation, 6 currently have protective titres. Minor side effects occurred after 11 vaccine doses in 9 children. No child developed varicella, despite 10 clear episodes of exposure to the wild-type virus.

Conclusions-Varicella immunisation in children with end stage and pre-end stage renal failure results in a high rate of seroconversion and persistence of protective antibody titres. More widespread use of the vaccine before renal transplantation is recommended.
\end{abstract}

(Arch Dis Child 2000;82:141-143)

Keywords: immunisation; varicella; end stage kidney failure; renal transplantation

Varicella in the immunosuppressed renal transplant recipient is a more serious infection than that observed in normal children, with considerable morbidity and a mortality rate of up to $25 \% \cdot{ }^{1-3}$ Affected children require admission to hospital and the use of high dose antiviral agents, which can impair allograft function. The development of varicella in those with no naturally acquired immunity can be prevented by the administration of zoster immune globulin soon after exposure to varicella zoster virus $(\mathrm{VZV})$ where this is recognised. Such treatment is expensive, involves intramuscular injection, and exposes the child to the risk of blood borne virus infection. Furthermore, passively acquired immunity is short lived, with further doses being required after subsequent exposure, and zoster immune globulin is in increasingly short supply. Although controversy surrounds universal childhood vaccina- tion against VZV, there is support for the immunisation of high risk populations. ${ }^{45}$

Current guidelines recommend that all children with pre-end stage and end stage renal failure receive all routine childhood immunisations and additional immunisations against hepatitis B, influenza, and Streptococcus pneumoniae. All necessary live immunisations should be administered before entry on to the transplantation waiting list because they are contraindicated once immunosuppressive treatment has been commenced. ${ }^{6}$ However, the presence of end stage renal failure is reported to reduce the immunological response to certain vaccines, including hepatitis B vaccine. ${ }^{7}$

The addition of live attenuated varicella vaccine to the above list to provide protective immunity after transplantation would be a logical step, although the subject has received little attention to date. A retrospective review of 212 children, who received a single 1000 plaque forming unit (PFU) dose of vaccine, reported an $87 \%$ seroconversion rate. Only $62 \%$ of children had protective antibody titres one year after immunisation, which fell to $52 \%$ at the time of transplantation. Despite the seemingly poor persistence of circulating antibody titres using this immunisation regimen, the incidence of varicella in the transplanted population fell from $45 \%$ to $12 \%$ after the introduction of immunisation. ${ }^{8}$ In a further study of 17 patients with end stage renal failure and 17 with functioning transplants who were also immunised with a single 1000 PFU dose of vaccine, $85 \%$ developed antibodies within the first six months, and $76 \%$ of the end stage patients had protective antibody titres at one year. Three children acquired varicella two to four years after successful immunisation, although in all cases the disease was in an attenuated form. ${ }^{9}$ Neither study reported data regarding exposure to wild-type VZV, and vaccine response rates were inferior to those reported elsewhere in healthy children. ${ }^{10}{ }^{11}$

We designed our study to determine whether immunisation of children with end stage and pre-end stage renal failure using a higher dose live attenuated varicella vaccine (two doses of 2000 PFU) resulted in an improved duration of immunity, and to collect prospective data regarding exposure to wild-type virus after immunisation. Our specific aims were: (1) to determine the seroconversion rate, duration of persistence of protective antibody titres, and incidence of side effects after immunisation; and (2) to determine the protective efficacy of this vaccine after seroconversion in those children subsequently exposed to VZV. 
Table 1 Primary renal diagnosis in study participants

\begin{tabular}{ll}
\hline Diagnosis & $n$ \\
\hline Renal dysplasia/hypoplasia & 10 \\
Congenital nephrotic syndrome & 5 \\
Nephronophthiasis & 3 \\
Posterior urethral valves & 3 \\
Prune belly syndrome & 2 \\
Other urological abnormalities & 2 \\
Bardet Biedel syndrome & 1 \\
Focal segmental glomerulosclerosis & 1 \\
Unspecified glomerulonephritis & 1 \\
Haemolytic uraemic syndrome & 1 \\
Interstitial nephritis & 1 \\
Reflux nephropathy & 1 \\
Towns Buck syndrome & 1 \\
\hline
\end{tabular}

\section{Methods}

The study group consisted of children with end stage renal failure on dialysis and pre-end stage renal failure (predicted glomerular filtration rate, $<20 \mathrm{ml} / \mathrm{min} / 1.73 \mathrm{~m}^{2}$ calculated using the Schwartz formula ${ }^{12}$ ) who had no previous history of varicella infection and were VZV IgG antibody negative by enzyme linked immunosorbent assay (ELISA). Children receiving immunosuppressive treatment were excluded from our study. Children were immunised using $2 \times 2000$ PFU doses of live attenuated varicella vaccine (Oka strain) (Pasteur Merieux MSD, Maidenhead, Berks UK) given three months apart. Serum VZV IgG antibody titres were measured by ELISA (Diamedix, Miami, USA), monthly for the first six months after immunisation and three monthly thereafter. Immunity to VZV was defined as a circulating antibody titre of $20 \mathrm{eu} / \mathrm{ml}$ or greater. ${ }^{13}$ At the time of immunisation, parents were issued with booklets to record any side effects and to document subsequent episodes of exposure to wild-type VZV. Where such exposure was recognised, the antibody titre was repeated and regular telephone contact was made with the family over the next 21 days. Where infection was suspected, children were reviewed by a principal investigator. Children awaiting renal transplantation were removed from the transplant waiting list for a six week period after each vaccine dose.

Fully informed consent was obtained from parents before entry into the study, for which ethical approval was granted by the Salford and Trafford, Royal Liverpool Children's Hospital, and St James's University Hospital local research ethics committees.

\section{Results}

A total of 32 children (20 boys, 12 girls) in the three participating centres were found to meet the study entry criteria, and all were entered into our study at a mean (SD) age at the time of first immunisation of 3.8 (2.9) years. Twenty five had end stage renal failure (19 on peritoneal dialysis and six on haemodialysis) and seven had pre-end stage renal failure; the range of primary renal pathologies is shown in table 1 . Thirty one children received both vaccine doses, and mean (SD) follow up from immunisation to date or to death was 19.1 (5.5) months. Four children have died, three after the elective withdrawal of end stage renal failure treatment (one between the first and second immunisations) and one after complications of transplantation. All 28 surviving children have completed at least one year of follow up after immunisation.

All children initially seroconverted after immunisation, and at a mean (SD) follow up of 20.3 (4.3) months, 23 of the 28 survivors had protective antibody titres (mean (SD) antibody titre, $71.8(47.5) \mathrm{eu} / \mathrm{ml}$ ). All four children who died had protective titres at the time of their death. Two children, both with end stage renal failure (one on peritoneal dialysis and one on haemodialysis) have required a third booster dose of vaccine, having initially seroconverted after immunisation but subsequently lost their protective antibody titre. After reimmunisation, both had protective antibody titres at 12 and eight months after their third vaccination.

Eleven children have undergone renal transplantation, of which 10 are currently alive. Seven are receiving cyclosporin A, azathioprine, and prednisolone, and three are receiving tacrolimus, azathioprine, and prednisolone immunosuppressive regimens. No child has required monoclonal or polyclonal antilymphocyte antibodies as induction or antirejection treatment. These 10 children have now been followed for a mean (SD) of 23.4 (3.9) months after immunisation and 14.2 (5.4) months after transplantation, and six currently have protective antibody titres. Ten of the 11 had protective titres at the time of transplantation. There have been no graft losses in this group, and the acute rejection rate of $30 \%$ is similar to that reported in most current paediatric series. ${ }^{14}$

A total of five children do not currently have protective antibody titres. Four of these have undergone transplantation, thus precluding the administration of a third booster dose, and the fifth is currently awaiting a booster dose. Of these four transplanted children, three had protective antibody titres at the time of transplantation, these being lost at two, 12 , and 13 months after transplantation. Overall, the characteristics of these five children did not

Table 2 Characteristics of the five children without current protective antibody titres

\begin{tabular}{|c|c|c|c|c|}
\hline Age at immunisation & $\operatorname{Sex}$ & Diagnosis & $\begin{array}{l}\text { Time post-immunisation of loss } \\
\text { of protective antibody titre }\end{array}$ & Current status \\
\hline 1 year 5 months & $\mathrm{F}$ & $\begin{array}{l}\text { Congenital nephrotic syndrome } \\
\text { (nephrectomised) }\end{array}$ & 4 months & Functioning transplant \\
\hline 4 years 0 months & $\mathrm{F}$ & Renal dysplasia & 1 year 10 months & Functioning transplant \\
\hline 2 years 1 month & M & Renal dysplasia & 2 years 0 months & Functioning transplant \\
\hline 6 years 9 months & $M$ & Posterior urethral valves & 2 years 0 months & Functioning transplant \\
\hline 2 years 11 months & $M$ & Renal dysplasia & 9 months & $\begin{array}{l}\text { Pre-end stage renal } \\
\text { failure }\end{array}$ \\
\hline
\end{tabular}


differ from the study population as a whole (table 2).

No major side effects were reported by parents after immunisation, although minor side effects that did not result in medical consultation occurred after 11 vaccine doses in nine children (fever, four; inflammation at immunisation site, seven). No child has developed zoster as a consequence of vaccination.

No child developed varicella, despite 10 clear episodes of exposure to wild-type VZV in a total of seven children, five before transplantation and five after transplantation. All children had satisfactory circulating antibody titres at the time of these contacts and remained clinically well.

\section{Discussion}

We have shown that immunisation with $2 \times 2000$ PFU doses of live attenuated varicella vaccine results in high levels of seroconversion and persistence of protective antibody titres in children with end stage and pre-end stage renal failure. These results are highly favourable compared with previous studies investigating varicella immunisation in children with renal failure, which reported $62 \%{ }^{8}$ and $76 \%{ }^{9}$ of children as having protective antibody titres at one year after immunisation. This might be because of the use of a higher vaccine dose and a two dose immunisation schedule. Recent evidence suggests that low dose vaccines are a risk factor for breakthrough varicella infection. ${ }^{15}$

Only two children required a third booster dose of vaccine after the loss of an initially protective antibody titre (a third is currently awaiting re-immunisation), and in both instances protective antibody titres were once again induced. Booster doses were only administered where children had not undergone transplantation. Current UK recommendations state that live vaccines should not be given to immunosuppressed solid organ transplant recipients, ${ }^{6}$ although there are reports of the use of varicella vaccine in this population. ${ }^{9}$ Immunisation before transplantation resulted in 10 of 11 children having protective antibody titres at the time of transplantation, and of the three children who subsequently lost their protective titres, two had immunity for the duration of the first 12 months after engraftment, when immunosuppressive treatment is most intense. We could deduce no obvious risk factors for the loss of protective antibody titres; no child had heavy proteinuria with consequent high urinary IgG losses. Although children with subprotective antibody titres were defined as being non-immune for the purposes of our study, cell mediated immune mechanisms might well have conferred satisfactory immunity.

There were no documented episodes of varicella infection after immunisation, despite a number of well documented episodes of exposure to wild-type VZV. The incidence of side effects was low, in keeping with other studies of this vaccine.

There are, at present, no UK or USA guidelines for the use of varicella vaccine in end stage and pre-end stage renal failure, although over $70 \%$ of North American nephrology centres recently surveyed stated that they recommended its use. ${ }^{16}$ The results of our study allow us to make a preliminary recommendation for the use of the vaccine in this population of children, using a $2 \times 2000 \mathrm{PFU}$ dose regimen before renal transplantation. Concentrations of anti-VZV IgG should be monitored on a regular basis after immunisation, and where loss of a previously protective antibody titre occurs, we recommend the use of a third booster dose, although we have not investigated this in children who have undergone transplantation in the intervening period. Larger numbers and a longer duration of follow up are clearly necessary before a more definite statement can be made, and further data need to be collected regarding the use of the vaccine in nonimmune patients who have already undergone transplantation. The vaccine also warrants further investigation in other at risk groups, including children with other end stage organ diseases likely to require solid organ transplantation, and those on long term continuous or intermittent immunosuppressive treatment for disorders such as childhood nephrotic syndrome.

Varicella vaccines for use in this study were kindly donated by Pasteur Merieux MSD. The authors would like to thank the parent of one of the subjects, for her generous donation, which allowed the funding of varicella zoster virus IgG enzyme linked immunosorbent assays.

1 Feldhoff CM, Balfour HH, Jr, Simmons RL, Najarian J, Mauer M. Varicella in children with renal transplants. $\mathcal{F}$ Pediatr 1981;98:25.

2 Lynfield R, Herrin JT, Rubin RH. Varicella in pediatric renal transplant patients. Pediatrics 1992;90:216-20.

3 Jaeggi A, Zurbruegg RP, Aebi C. Complications of varicella in a defined central European population. Arch Dis Child 1998;79:472-7.

4 Friedman Ross L, Lantos JD. Immunisation against chickenpox. BMF 1995;310:2-3.

5 Gershon A. Varicella: to vaccinate or not to vaccinate. Arch Dis Child 1998;79:470-1.

6 Department of Health. Immunisation against infectious disease. London: HMSO, 1996.

7 Pasko MT, Pharm D, Bartholomew WR, Beam TR, Amsterdam D, Cunningham EE. Long term evaluation of hepatitis B vaccine (Heptavax-B) in haemodialysis patients. Am f Kidney Dis 1988;11:326-31.

Am F Kidney Dis 1988;11:326-31.
8 Broyer M, Tete MJ, Guest G, Gagnadoux MF, Rouzioux C. Varicella and zoster in children after kidney transplantation: long-term results of vaccination. Pediatrics 1997;99:35-9.

9 Zamora I, Simon JM, Da Silva ME, Piqueras AI. Attenuated varicella virus vaccine in children with renal transplants. Pediatr Nephrol 1994;8:190-2.

10 Watson B, Gupta R, Randall T, Starr S. Persistence of cellmediated and humoral immune responses in healthy children immunised with live attenuated varicella virus. $\mathcal{F}$ Infect Dis 1994;164:197-9.

1 Asano Y, Nagai T, Miyata T, et al. Long term protective immunity of recipients of the Oka strain of varicella vaccine. Pediatrics 1985;75:667-71.

12 Schwartz GJ, Haycock G, Edelman C, Spitzer A. A simple estimate of glomerular filtration rate in children derived from body length and plasma creatinine. Pediatrics 1976;58:259-63.

13 Shehab Z, Brunell PA. Enzyme-linked immunosorbent assay for susceptibility to varicella. F Infect Dis 1983;148: 472-6.

14 Kohaut EC, Tejani A. The 1994 annual report of the North American pediatric renal transplant cooperative study. Pediatr Nephrol 1996;10:422-34.

15 Lim YJ, Chew FT, Tan AYS, Lee BW. Risk factors for breakthrough varicella in healthy children. Arch Dis Child 1998;79:478-80

16 Furth SL, Neu AM, Sullivan K, Gensler G, Tejani A, Fivush BA. Immunisation practices in children with renal disease: a report of the North American paediatric renal transplant cooperative study. Pediatr Nephrol 1997;11:443-6. 